\title{
DEDDWTE

\section{5) Avaliação de fatores de risco para colonização por microrganismos multirresistentes}

\author{
Lidiane Riva Pagnussat ${ }^{1}$; Adriano Pasqualotti ${ }^{2}$
}

\section{Resumo}

O aumento das bactérias multirresistentes (MDR) e a necessidade de minimizar possíveis transmissões cruzadas ressaltam a importância de medidas de controle de disseminação desses patógenos, entre as quais o rastreamento de MDR em paciente com fatores de risco. O objetivo foi identificar a colonização de MDR em pacientes institucionalizados no momento da internação. O estudo é do tipo retrospectivo e descritivo, realizado em hospital de ensino de alta complexidade. Analisou-se os resultados dos swabs de vigilância realizados no período de janeiro a junho de 2018 em pacientes que possuíam ao menos um fator de risco para MDR. Foram avaliados 863 pacientes, sendo que 45 (5,2\%) eram institucionalizados e destes $4(8,9 \%)$ apresentaram amostra colonizada por MDR. Esses parâmetros demonstram a importância do rastreamento e controle de disseminação de microrganismos em ambiente hospitalar.

Palavras-chaves: Resistência microbiana a medicamentos; Controle de infecção; Institucionalização

\section{As infecções associadas aos cuidados de saúde}

O envelhecimento da população é uma das mais significativas tendências do século XXI. As projeções e estimativas da população do Brasil indicam que a população de pessoas com 65 anos ou mais passou de 7,3\% em 2010 para 8,9\% em 2017. Estima-se que chegará a 25,2\% em 2058 (IBGE, 2018). Este fenômeno aumenta as necessidades de cuidados à saúde e a demanda assistencial à população. Contrário a esta população, o número de pessoas potencialmente cuidadoras, com idades entre 50 e 60 anos, está diminuído. Estima-se que em 2050 haverá apenas dois cuidadores para cada idoso de 75 anos ou mais. Esses fatores aumentaram a procura pelos serviços de institucionalização para idosos que precisam de cuidados diários e de longa duração (CAMARGOS, 2014).

1 Mestranda no Programa de Pós-Graduação em Envelhecimento Humano, Faculdade de Educação Física e Fisioterapia, Universidade de Passo Fundo. Endereço para correspondência: Universidade de Passo Fundo, Campus I, BR 285, Bairro São José, CEP 99052-900, Passo Fundo, Rio Grande do Sul, Brasil. Email: lidianerpagnussat@gmail.com

2 Professor do Programa de Pós-Graduação em Envelhecimento Humano, Instituto de Ciências Exatas e Geociências Universidade de Passo Fundo. Email: pasqualotti@upf.br 
O número de comorbidades dos pacientes institucionalizados, o compartilhamento de espaços e objetos comuns, o contato com os profissionais da saúde e cuidadores, associado a possíveis falhas de controle de infecção e a utilização de antibióticos são fatores de risco que contribuem para a colonização por microrganismos multirresistentes. As infecções associadas aos cuidados de saúde são consideradas um problema difícil de controle, que aumentam a gravidade da infecção, a necessidade de internação e podem levar a morte (CDC, 2013). Os microrganismos resistentes às diversas classes de antimicrobiano tem sido progressiva nas últimas décadas. Atualmente é considerada uma ameaça à saúde pública em todo mundo. Estima que, anualmente, pelo menos dois milhões de infecções e 23 mil mortes são causadas por bactérias resistentes aos antibióticos nos Estados Unidos. A principal causa é o uso indiscriminado e inadequado dos antimicrobianos (BRASIL, 2017).

As consequências diretas das infecções causadas por microrganismos resistentes aos antimicrobianos são graves. Incluem o aumento da morbidade e mortalidade, a necessidade de internação hospitalar e aumento desse período, a redução ou perda da proteção para os pacientes (alterações na flora normal) submetidos a procedimentos de cirurgia, quimioterapia e transplantes, redução do arsenal tecnológico ou a falta de opção terapêutica (antibióticos que inibam o crescimento ou provocam sua morte) para o tratamento diante de alguns microrganismos causadores da infecção e o aumento dos custos de tratamento e internação. Estima-se que nos Estados Unidos o custo com resistência bacteriana é de aproximadamente 5 bilhões de dólares anualmente (BRASIL, 2017). Para enfrentar esse problema é necessário inúmeras ferramentas e estratégias: i) investimentos a longo prazo que fomentam o desenvolvimento de novos medicamentos; ii) ferramentas de diagnóstico, vacinas e outras intervenções; iii) fortalecimento dos sistemas de saúde a fim de promover o acesso e a utilização eficaz de agentes antimicrobianos; iv) implantação de rotinas de controle de infecção em todas as instituições de saúde, como por exemplo, higienização das mãos, limpeza do ambiente (BRASIL, 2017).

Uma pesquisa realizada em São Paulo identificou 166 casos de infecção por microrganismos multidroga resistentes de início na comunidade. Os fatores de risco associados foram doença pulmonar e internação prévia, sendo que para bactérias gram-negativos a idade também foi considerada. $\mathrm{O}$ estudo mostrou a possibilidade de transmissão de microrganismos MDR em ambientes comunitários ou serviços de saúde não hospitalares (PEREIRA, 2018). Outra pesquisa realizada em São Paulo com pessoas residentes em instituições de longa permanência analisou 62 amostras nasais em 62 idosos, sendo que 39 institucionalizados de um a oito anos e sete com mais de 25 anos. Os resultados demostraram que das amostras coletadas, cinco foram positivas para Staphylococcus aureus resistentes a meticilina (MRSA). Sendo que dessas, $80 \%$ usaram sondas e antibióticos e $20 \%$ passaram por atendimento hospitalar e internação (FLORENTINO, 2018). Outro estudo realizado com 1.537 pacientes internados em um hospital da cidade de Montenegro/RS, apontou que 8,1\% apresentaram cultura positiva para bactéria multirresistente e 10,9\% indicaram positivo nos swabs de vigilância. Os fatores de risco para MDR identificados nas culturas de $s w a b$ de vigilância foram: internação superior a sete dias (OR: 2,24; $\mathrm{IC}_{95 \%}[2,0 ; 5,2] ; \mathrm{p}<0,001$ ); necessidade do uso de antibiótico (OR: 2,73; $\left.\mathrm{IC}_{95 \%}[1,3 ; 5,6] ; \mathrm{p}=0,010\right)$; paciente oriundo de instituição geriátrica (OR: 6,$79 ; \mathrm{IC}_{95 \%}[2,9$; 
$15,9] ; \mathrm{p}=0,001) ;$ paciente transferido de outro hospital (OR: 5,55; $\mathrm{IC}_{95 \%}[2,1 ; 14,4] ; \mathrm{p}=0,001$ ) (ROHR, 2014).

A detecção precoce de pacientes colonizados por microrganismos multirresistentes, realizada por culturas de vigilância epidemiológica e a implementação de medidas preventivas pode reduzir a incidência destes microrganismos. $\mathrm{O}$ objetivo deste trabalho foi identificar o percentual de pacientes institucionalizados colonizados por microrganismos multirresistentes no momento da internação.

\section{Material e métodos}

O estudo é do tipo retrospectivo e descritivo, realizado em um hospital de ensino de alta complexidade, localizado no norte do estado do Rio Grande do Sul. O hospital é considerado uma unidade de referência em saúde para municípios da região Sul do Brasil. Os dados analisados fazem parte do banco de dados do Serviço de Controle de Infecção Hospitalar (SCIH). Foram analisados os resultados dos swabs de vigilância solicitados pelo SCIH. Os tipos analisados foram axilar, inguinal, testa e $s w a b$ retal específico para pesquisa de enterobactérias resistente a carbapenêmicos. A coleta foi realizada no período de janeiro a junho de 2018 .

Os swabs foram realizados em pacientes que internaram nas primeiras 72 horas de internação e que possuíam ao menos um desses fatores de risco para bactéria multirresistente: i) internação em outros hospitais por mais de 72 horas; ii) institucionalizados que realizaram procedimento invasivo em outra instituição no último mês; iii); internação em unidade de terapia intensiva de outros hospitais nos últimos 6 meses; e iv) contatos de outros pacientes que tiveram culturas de bactérias multirresistentes.

A instituição considera bactéria multirresistente as bactérias gram-negativas (Acinetobacter sp., Pseudomonas sp. e enterobactérias) resistentes a carbapenêmicos e as gram-positivas (Enterococcus sp. e Staphylococcus) resistentes à vancomicina.

\section{Resultados}

Foram avaliados 863 pacientes, sendo a maioria do sexo masculino (56\%). Foram realizados 855 swabs retais e destes 66 (7,7\%) tiveram crescimento de enterobactérias resistentes a carbapenêmicos. Realizou-se 453 swabs axilares, sendo que sete (1,5\%) apontaram crescimento de MDR); 452 swabs de testa, sendo que 4 (0,9\%) indicaram crescimento de MDR; e 452 swabs inguinais, sendo que 19 (4,2\%) apontaram crescimento de MDR. Entre os pacientes avaliados apenas $79(9,0 \%)$ foram identificados como colonizados por bactérias resistentes a carbapenêmicos nos swabs de vigilância, sendo que destes pacientes colonizados $27(34,1 \%)$ tinham internação prévia em outro hospital, 4 (5,1\%) eram institucionalizados, $47(59,5 \%)$ eram contatos de outros pacientes com bactéria multirresistente. Não houve crescimento de bactérias gram-positivas resistentes à vancomicina nos swabs realizados. Dos 79 pacientes colonizados por MDR, apenas três apresentaram amostras clínicas com crescimento de MDR. Do total de pacientes avaliados, $45(5,2 \%)$ pacientes eram institucionalizados. Nesses pacientes foram realizados 45 swabs retais e foram identificadas $3(6,7 \%)$ culturas positivas para Kle- 
bsiella pneumoniae resistente a carbapenêmicos; 41 swabs de axila com uma $(2,2 \%)$ cultura positiva para Escherichia coli multirresistente; 41 swabs de testa apontaram desenvolvimento de MDR e outros 41 swabs inguinais com $3(6,7 \%)$ culturas positivas para MDR (uma para Escherichia coli, 1uma para Burkholderia sp e outra para Klebsiella pneumoniae resistentes a carbapenêmicos). No total, 4 (8,9\%) pacientes estavam colonizados por MDR. Avaliando as culturas clínicas dos 45 pacientes institucionalizados, três tiveram culturas clínicas com crescimento de bactérias resistentes a carbapenêmicos, sendo que dois desses pacientes com cultura de aspirado traqueal positiva para Acinetobacter sp. e outro com uroculturas positivas para Enterobacter e Klebsiella pneumoniae). Nenhum destes pacientes foram identificados pelos swabs de vigilância epidemiológica. Somando os pacientes colonizados (identificados pelos swabs) com os pacientes com amostras clínicas positivas, 7 (15,6\%) dos pacientes institucionalizados tiveram culturas positivas para MDR.

Os resultados encontrados corroboram sobre a necessidade de intervenções efetivas no ambiente hospitalar para minimizar o problema da resistência microbiana. As estratégias de vigilância capazes de realizar identificação precoce de bactérias multirresistentes estão entre os componentes recomendados pela Agencia Nacional de Vigilância Sanitária (ANVISA), que sugere ainda a investigação e controle de surtos, protocolos de esterilização e desinfecção de equipamentos, além da implementação de práticas de cuidados ao paciente, tais como higienização das mãos, isolamento e barreiras entre pacientes infectados/colonizados, bem como a implantação de programas efetivos de controle do uso de antimicrobianos, pois o uso destes é, provavelmente, a principal causa de resistência bacteriana (BRASIL, 2017).

A implementação de programas intensivos de prevenção e controle de infecção relacionada aos cuidados de saúde consiste numa abordagem eficiente para o controle da disseminação da resistência microbiana no ambiente hospitalar. Entretanto, os resultados da pesquisa mostram a importância de estender esses programas para todas as instituições de saúde não hospitalares. Esses ambientes podem ser uma fonte importante de disseminação destes microrganismos, principalmente porque a complexidade e a presença de doenças crônicas nesses pacientes levam a uma certa dependência e necessidade de internação hospitalar, realização de procedimentos e tratamento de infecções frequentes em diferentes hospitais e clínicas (BRASIL, 2017).

\section{Conclusões}

A realização de swabs de vigilância contribui no rastreamento e controle de disseminação de microrganismos no ambiente hospitalar. A institucionalização pode ser um fator de risco para a colonização de MDR. As medidas de controle de infecção, principalmente relacionadas a limpeza do ambiente e higienização das mãos, e o uso racional de antimicrobianos devem ser implementadas em todas as instituições de saúde hospitalares e não hospitalares. Esses fatores de risco podem estar contribuindo na disseminação destes microrganismos. A implementação de programas intensivos de prevenção e controle de infecção relacionada aos cuidados de saúde são eficientes para o controle da disseminação da resistência microbiana. 


\section{Evaluation of risk factors for colonization by multiresistant microorganisms}

\section{Abstract}

The increase of multidrug resistant bacteria (MDR) and the need to minimize possible cross-transmissions underscore the importance of measures to control the spread of these pathogens, including MDR screening in patients with risk factors. The objective was to identify the colonization of MDR in institutionalized patients at the time of hospitalization. The study is of the retrospective and descriptive type, carried out in a teaching hospital of high complexity. The results of the surveillance swabs performed in the period from January to June of 2018 were analyzed in patients who had at least one risk factor for MDR. A total of 863 patients were evaluated, of which $45(5.2 \%)$ were institutionalized and of these $4(8.9 \%)$ had a sample colonized by MDR. These parameters demonstrate the importance of tracking and controlling the spread of microorganisms in a hospital environment.

Keywords: Microbial resistance to drugs; Infection control; Institutionalization

\section{Referências}

BRASIL. Agência Nacional de Vigilância Sanitária. Plano Nacional para a Prevenção e o Controle da Resistência Microbiana nos Serviços de Saúde. Brasília, 15 de maio de 2017.

CAMARGOS, M. C. S. Instituições de longa permanência para idosos: um estudo sobre a necessidade de vagas. Rev. Bras. Estud. Popul., São Paulo, v. 31, n. 1, p. 211-217, jun. 2014.

CDC. Antibiotic resistance threats in the United States, 2013 US Department of Health and Human Services. CDC, 2013. Disponível em: <http://www.cdc.gov/drugresistance/threat-report-2013/index.html>. Acesso em: 10 set. 2018.

FLORENTINO, A. O. Staphylococcus aureus resistente à meticilina: avaliação em Instituições de longa permanência para idosos. 2018. f. 76. Dissertação (Mestrado) - Faculdade de Medicina de Botucatu, Universidade Estadual Paulista "Júlio de Mesquita Filho"; 2018.

IBGE. Instituto Brasileiro de Geografia e Estatística. Projeções e estimativas da população do Brasil. Disponível em: https://www.ibge.gov.br/apps/populacao/projecao/. Acesso em: 10 set. 2018.

PEREIRA, M. A. D. Microrganismos multidroga-resistentes na fronteira entre serviços de saúde e comunidade: uma abordagem espacial. 2018. f. 67. Dissertação (Mestrado) - Faculdade de Medicina de Botucatu, Universidade Estadual Paulista Júlio de Mesquita Filho"; 2018.

ROHR, D. et al. Fatores associados à infecção ou contaminação por germe multirresistente em pacientes clínicos de hospital secundário do interior do Brasil 100\% SUS. São Paulo: Blucher, 2014. 